\title{
Research progress in control method of water treatment membrane fouling
}

\author{
WANG Hanbin ${ }^{1,}$,, ZHANG Dehua ${ }^{2, b}$ \\ ${ }^{1,2}$ School of environmental science \& engineering, Kunming University of Science and Technology, \\ Yunnan Kunming, 650000 \\ aemail:whb0070@126.com, ${ }^{\mathrm{b}}$ email:kmzdh@sina.com
}

Keywords:membrane fouling; morphology and structure; hydrophobic; charge; membrane coupling process

Abstract. The research progress of membrane fouling control method in water treatment is introduced by four aspects of solution environment, hydraulics condition, membrane property and strengthening coupling process. Former two influence membrane fouling process by changing system charge and membrane surface shear stress. Modified anti-fouling membrane could be achieved by membrane structure improvement, hydrophilic modification and membrane charge modification. The modified method can be divided into casting solution modification and membrane surface modification. Point out that the research of membrane fouling on-line control method should be focus on the preparation of high performance modifiers.

\section{Membrane fouling mechanism}

In the process of osmotic solution formation by filtrating solvent, concentration of pollution near the membrane surface is increased and the concentration polarization is formed; contaminants adsorption and blocking of membrane pores on the membrane surface formed irreversible membrane fouling. In the process of filtering the solution, the above three kinds of conditions will cause the increase of membrane resistance and flux decline with the increase of the filtration time. Membrane fouling was decided by electrostatic forces, hydrogen bonding, dipole moment and Kulun force between contaminant and membrane; pollutant charge effect and size effect are two main factors that affect membrane fouling.

\section{Control method of membrane pollution}

Improve the solution environment. Solution $\mathrm{pH}$ affects the charge characteristics of the solute and the membrane, thus changing the adhesion force between them, which further affects the membrane fouling process; Meng Xiaorong[1] et al. proved the above viewpoint by means of atomic force test on humic acid and membrane under different $\mathrm{pH}$ conditions. In single solute system, Membrane pollution is the most serious when the $\mathrm{pH}$ is at the isoelectric point of the solute; In the solute system of two elements, when $\mathrm{pH}$ is in between the isoelectric point, due to the charge attraction between the solutes to form a stable dynamic equilibrium, the membrane fouling is more serious than that of the single system and is less affected by $\mathrm{pH}$. In addition, the carboxyl group in the molecule under low $\mathrm{pH}$ condition is hindered, the charge property of the molecule and the repulsive force between the solute and the membrane were reduced, which makes the membrane fouling more easily.

The composition of solution can also affect the membrane fouling in different degrees. The influence of organic matter composition on membrane fouling was studied by using four kinds of different sources of humic acid and different molecular weight cut of ultrafiltration membrane; The results showed that the contribution rate of membrane fouling resistance to total resistance was decreased when the membrane fouling rate of humic acid was more than $40 \%$, and the contribution of the membrane itself resistance to total membrane resistance increased with the increase of the rejection rate. When the initial solution concentration is higher, the membrane flux decay rate increases, but does not necessarily affect the equilibrium flux. The inorganic ions in the solution can affect the membrane fouling process by changing the charged properties of the organic matter, the double layer of the 
compressed solute and the generation of the complex reaction; Among them, two valence cations $\left(\mathrm{Ca}^{2+}\right.$, $\mathrm{Mg}^{2+}$ ion, etc.) are generally considered to have a great influence on membrane fouling. $\mathrm{Ca}^{2+}$ can combined with organic matter by bridge formation and increase the fouling [2].

Improving fluid mechanics. Because the shear stress can be produced on the surface of the membrane and the pollution layer on the membrane surface can be washed, membrane fouling is relatively mild in cross flow filtration compared with the dead end filtration. The irreversible membrane fouling caused by constant pressure filtration is more serious than that by the constant pressure filtration in the long run, because the constant pressure generally requires high pressure conditions, and higher pressure can increase the rate of fouling. In the pretreatment of coagulation - microfiltration membrane separation process, the high membrane surface flow rate increases the pollution of the filter, but it can restrain the formation of cake layer, which is suitable for long term operation.

For microfiltration, ultrafiltration process of pore flow model, in pressure control water flux of membrane region increases with the increase of operating pressure, when the pressure increases to non pressure control zone, increasing the operation pressure is on the pure water flux of the membrane had no effect, and some studies show that increasing the operating pressure can accelerate the membrane fouling.

Improve the properties of the membrane. Membrane antifouling property can be improved by structure, hydrophilic and charge modification. Commonly used structural modifiers include polyethylene glycol (PEG), polyvinyl chloride (PVP) and chlorinated ( $\mathrm{LiCl}$ ); Negatively charged modifiers usually containing sulfonic acid group and carboxyl group, positively charged modifiers usually containing quaternary ammonium salt and phosphine; Hydrophilic modification agent is usually containing sulfonic acid group $\left(-\mathrm{SO}_{3}\right)$, carboxyl $(-\mathrm{COOH})$, hydroxyl $(-\mathrm{OH})$ and other groups of amphiphilic block copolymer, hydrophilic monomer and its esters, or $\mathrm{TiO}_{2}, \mathrm{SiO}_{2}, \mathrm{ZrO}_{2}$ and other inorganic nanoparticles.

Improvement of membrane structure. The basic structure of the membrane (such as spongy pore, finger pore, thickness, etc.), membrane pore characteristics (porosity, pore size, pore size distribution) and the surface roughness of the membrane will have an impact on the degree of membrane fouling. The basic structure and roughness of the membrane were observed by scanning electron microscopy (SEM) and atomic force microscopy (AFM). The membrane pores were characterized by weight method, bubble pressure method, mercury injection method and so on. In addition, the influence of the molecular brush structure of the membrane surface on the antifoulingproperty should be taken into account.

Some study showed that the surface morphology of the membrane treated with high concentration of food wastewater, the more rough of the membrane surface, the faster flux decay, and the more difficult flux recovery after chemical cleaning. Some researchers also studies the preparation of membrane pore structure for type $\mathrm{A}$ and type $\mathrm{V}$ two kinds of hollow fiber membrane, testing them on these typical ovalbumin, starch, $\mathrm{SiO}_{2}$ suspensions, diesel pollutants filtration performance, The results showed that the higher surface roughness $\mathrm{V}$ type mesoporous fiber membrane got a stronger antifouling property since it has a higher effective filtration area and is not easy to damage the surface of the membrane. Therefore, it is not only to judge the antifouling property of the membrane by the membrane surface structure parameters.

Improvement of membrane hydrophilicity. The hydrophilicity of the membrane is usually characterized by contact angle, and generally, the membrane has better antifouling property with stronger hydrophilicity. The membrane hydrophilic modification was carried out by three kends of methods: Blending, surface coating, surface grafting. the hydrophilic material used for blending method is limited, and have to consider the compatibility with the polymer,the surface modified by nanoparticles with hydrophilic groups will become a hot spot of research; The coating method is simple to operate, but the stability is poor; Grafting reaction condition is mild with stable graft functional group, and the amount of grafting is easy to control. Modified methods are difficult to innovate and breakthrough, the future research focus should be placed on the preparation of modified agent. 
Liu Dapeng[3] et al preparaed PMMA-b-PEG-b-PMMA amphiphilic hydrophilic modifier by Atom transfer radical polymerization at first, and then PVDF blending membranes were prepared by phase inversion method. PMMA hydrophobic segment embedded in the membrane, the hydrophilic segments PEG on the membrane surface in contact with the water, forming the terry structure. The contact angle is reduced from $95.9^{\circ}$ to $64.9^{\circ}$, pure water flux increased to $150 \%$, BSA permeate flux increased to $330 \%$, membrane cleaning flux recovery rate increased from $58 \%$ to $97 \%$ after hydrophilic modification.

Meng-Xin $\mathrm{Hu}[4]$ et al make The PVDF membrane immersed in amphiphilic cholic acid (CA) solution, the hydrophilic modification of PVDF membrane was carried out by the adsorption between the nonpolar part of the membrane and the CA. The hydrophilicity of the membrane was improved, and the water flux reached $200 \%$ of the unmodified membrane when the concentration of CA solution was above $0.088 \mathrm{~mol} / \mathrm{L}$. Lei Wang[5]et al preparaed 3- hydroxy -2,2- bis (hydroxymethyl) - acrylate (PEA) hydrophilic modifier by esterification reaction between the pentaerythritol (PE) and acrylic acid (AA) at first, and then PP-g-PEA hydrophilic membrane was prepared under UV continuous irradiation conditions. Drops of water added to the surface of the membrane can be lost within 6.3 seconds, the membrane pure water flux increased to 4.5 times.

Improvement of membrane charge. The charged properties of the membrane can be characterized by the zeta potential of the membrane surface. The most commonly used method is to sulfonated, and the commonly used sulfonated reagent is concentrated sulfuric acid and chlorine sulfonic acid. Xing Garry[6]et al use the chlorine sulfonic acid to carry on the sulfonated PVDF membrane, there is no essential change in the morphology of sulfonated charged membrane, the permeation flux and rejection rate of polystyrene sulfonic acid solution were all increased. Lixin[7]et al preare silica microspheres by sol gel method,then 2- bromo isobutyryl bromide embedded on the surface of silica,at last, $\mathrm{SiO}_{2}$-PSS modifier was prepared by ATRP method, and the $\mathrm{SiO}_{2}-\mathrm{PSS} / \mathrm{PES}$ blend membranes were prepared by phase inversion method, and the purified water flux was improved after modification. The introduction of acidic functional groups is easy to form water clusters to improve the hydrophilicity, which produce homogeneous repulsion between the charged membrane and suspended solids, reducing pollutant deposition on the membrane surface, the membrane antifouling property was improved. The researcher soaked the regenerated cellulose membrane in Sodium 3-bromopropanesulfonate solution to carry on charged modification, after modification the retention rate of humic acid increased from 59\% to $92 \%$, and the flux decline decreased from $32 \%$ to $25 \%$ after 4 hours of filtration.

Enhanced coupling process. Coagulation-membrane separation process:many studies have indicated that coagulation pretreatment or on-line coagulation can have a positive effect on the membrane separation process. The most immediate phenomenon is the removal of suspended solids in water by coagulation. For example: since ferric chloride was used for surface water treatment the turbidity reduced to below 5NTU, after coagulation, the size of the pollutants increased significantly, and the membrane fouling was reduced[8]. Polymeric aluminum chloride can reduce the reversible pollution; polyacrylamide forms the multiple curved boundaries and the lower effective density, to form a more loose and porous cake layer on the membrane surface, this leads to a reduction in both reversible and irreversible pollution ${ }^{[9]}$. There are studies show that membrane flux attenuation will be further reduced when organic coagulant dimethyl diallyl ammonium chloride Poly (PD) In coordination with the use of aluminum salt inorganic coagulant[10], and continuous dosing has better effect than Single reagent dosing[11]. The size and dimension of the flocculation and the amount of charge are the key factors influencing the membrane fouling.

Photocatalysis membrane separation coupling process:The problem has been widely studied by many scholars because it can simultaneously solve the optical catalyst loss, membrane pollution and Non complete degradation of pollutants in the membrane water treatment process[12]. Photocatalysis is a good way to reduce membrane fouling. however, under some conditions, some large molecules are degraded into small molecules and the rejection rate is reduced. 
$\mathrm{TiO}_{2}$ modified photo catalytic film will form more hydroxyl groups on the surface of the film under UV irradiation, to increase the hydrophilicity and to reduce the filter cake layer[13]; But too much $\mathrm{TiO}_{2}$ addition can Cause membrane pore blockage, flux decline, so the amount of $\mathrm{TiO}_{2}$ has a optimum value[14]. The adsorption of pollutants near the membrane surface and membrane pores will be degraded by the photocatalytic of $\mathrm{TiO}_{2}$. However, it is important to note that the membrane pore size is very important for the photocatalytic membrane separation process, the pollutants enter the inner membrane pores and the ultraviolet light transmittance becomes poor when the membrane hole is large, the photocatalytic degradation of pollutants will be blocked[15].

In addition, the coupling process such as electric field enhanced ultrafiltration, ultrasound enhanced ultrafiltration and aeration enhanced ultrafiltration were also widely studied to control membrane fouling.

\section{Conclusions}

In order to make membrane water treatment technology more widely used, and avoid the production of secondary wastewater, It is necessary to analyze, study and improve the method of on-line control of membrane pollution. The control of solution environment is easy to be realized under the current technical level; it is convenient and effective to design energy saving and efficiency membrane pollution control module and enhanced coupling process; to preparate antifouling membrane with excellent structure, hydrophilicity and charging performance is a fundamental method to control membrane fouling. In the research of membrane anti fouling modification, modified methods are difficult to innovate and breakthrough, research emphasis should be put on the synthesis of highly efficient modifiers.

\section{References}

[1] Meng Xiaorong, Tang Weiting, Wang Lei, et al. Mechanism analysis of membrane fouling behavior by humic acid using atomic force microscopy: Effect of solution $\mathrm{pH}$ and hydrophilicity of PVDF ultrafiltration membrane interface[J]. Journal of Membrane Science, 2015,487:180-188.

[2] Jermann D., Pronk W., Meylan S., et al. Interplay of different NOM fouling mechanisms during ultrafiltration for drinking water production[J]. Water Research, 2007,41(8):1713-22.

[3] Liu Dapeng, Li Dan, Du De, et al. Antifouling PVDF membrane with hydrophilic surface of terry pile-like structure[J]. Journal of Membrane Science, 2015,493:243-251.

[4] Hu M. X., Li J. N., Zhang S. L., et al. Hydrophilic modification of PVDF microfiltration membranes by adsorption of facial amphiphile cholic acid[J]. Colloids Surf B Biointerfaces, 2014,123:809-13.

[5] Wang Lei, Wei Junfu, Zhao Kongyin, et al. Preparation and characterization of high-hydrophilic polyhydroxy functional PP hollow fiber membrane[J]. Materials Letters, 2015,159:189-192.

[6] Baroña Garry Nathaniel B., Cha Bong Jun, Jung Bumsuk. Negatively charged poly(vinylidene fluoride) microfiltration membranes by sulfonation[J]. Journal of Membrane Science, 2007,290(1-2):46-54.

[7] Xing Lixin, Guo Ningning, Zhang Yatao, et al. A negatively charged loose nanofiltration membrane by blending with poly (sodium 4-styrene sulfonate) grafted $\mathrm{SiO}_{2}$ via SI-ATRP for dye purification[J]. Separation and Purification Technology, 2015,146:50-59.

[8] Choksuchart Porntip, Hean Marc, Grasmick Alain. Ultrafiltration enhanced by coagulation in an immersed membrane system[J]. Desalination, 2002,145:265-272. 
[9] Yao Meng, Nan Jun, Chen Ting, et al. Influence of flocs breakage process on membrane fouling in coagulation/ultrafiltration process - Effect of additional coagulant of poly-aluminum chloride and polyacrylamide[J]. Journal of Membrane Science, 2015,491:63-72.

[10] Xu Weiying, Yue Qinyan, Gao Baoyu, et al. Impacts of organic coagulant aid on purification performance and membrane fouling of coagulation/ultrafiltration hybrid process with different Al-based coagulants[J]. Desalination, 2015,363:126-133.

[11]Gong H., Jin Z., Wang X., et al. Membrane fouling controlled by coagulation/adsorption during direct sewage membrane filtration (DSMF) for organic matter concentration[J]. J Environ Sci (China), 2015,32:1-7.

[12]Leong Sookwan, Razmjou Amir, Wang Kun, et al. $\mathrm{TiO}_{2}$ based photocatalytic membranes: A review[J]. Journal of Membrane Science, 2014,472:167-184.

[13] Madaeni S. S., Zinadini S., Vatanpour V. A new approach to improve antifouling property of PVDF membrane using in situ polymerization of PAA functionalized $\mathrm{TiO}_{2}$ nanoparticles[J]. Journal of Membrane Science, 2011,380(1-2):155-162.

[14] You S. J., Semblante G. U., Lu S. C., et al. Evaluation of the antifouling and photocatalytic properties of poly(vinylidene fluoride) plasma-grafted poly(acrylic acid) membrane with self-assembled $\mathrm{TiO}_{2}$ [J]. Journal of Hazardous Materials, 2012,237-238:10-9.

[15]Damodar R. A., You S. J., Chou H. H. Study the self cleaning, antibacterial and photocatalytic properties of $\mathrm{TiO}_{2}$ entrapped PVDF membranes[J]. J Hazard Mater, 2009,172(2-3):1321-8. 